\title{
Search for High-Extinction Regions in the Small Magellanic Cloud
}

\author{
M.-Y. Lee ${ }^{1}$, S. Stanimirovići ${ }^{1}$, J. Ott ${ }^{2}$, J. Th. van Loon $^{3}$, \\ A. D. Bolatto ${ }^{4}$, P. A. Jones ${ }^{5,6}$, M. R. Cunningham ${ }^{5}$,
}

K. E. Devine $^{1}$ and J. M. Oliveira ${ }^{3}$

${ }^{1}$ Department of Astronomy, University of Wisconsin-Madison, USA email: lee@astro.wisc.edu ${ }^{2}$ National Radio Astronomy Observatory, ${ }^{3}$ Astrophysics Group, Keele University, ${ }^{4}$ Department of Astronomy, University of Maryland, ${ }^{5}$ School of Physics, University of New South Wales, ${ }^{6}$ Departamento de Astronomia, Universidad de Chile

We have applied the unsharp-masking technique to the $24 \mu \mathrm{m}$ image of the SMC, obtained with the Spitzer, to search for high-extinction regions. Fifty-five candidate regions of high-extincion (namely high-contrast regions, HCRs) have been identified from the decremental contrast image. HCRs have a size of $8-14 \mathrm{pc}$ and a peak contrast at $24 \mu \mathrm{m}$ of $2-2.5 \%$. To constrain physical properties of HCRs, we have performed observations of $\mathrm{NH}_{3}, \mathrm{~N}_{2} \mathrm{H}^{+}, \mathrm{HNC}, \mathrm{HCO}^{+}$, and HCN toward one of the HCRs, HCR LIRS36-east, using the ATCA and the Mopra telescope. No molecular line emission detected, but upper limits to column densities of molecular species suggest that HCRs are moderately dense with $n \sim 10^{3} \mathrm{~cm}^{-3}$. Two interesting properties of HCRs are shown below.
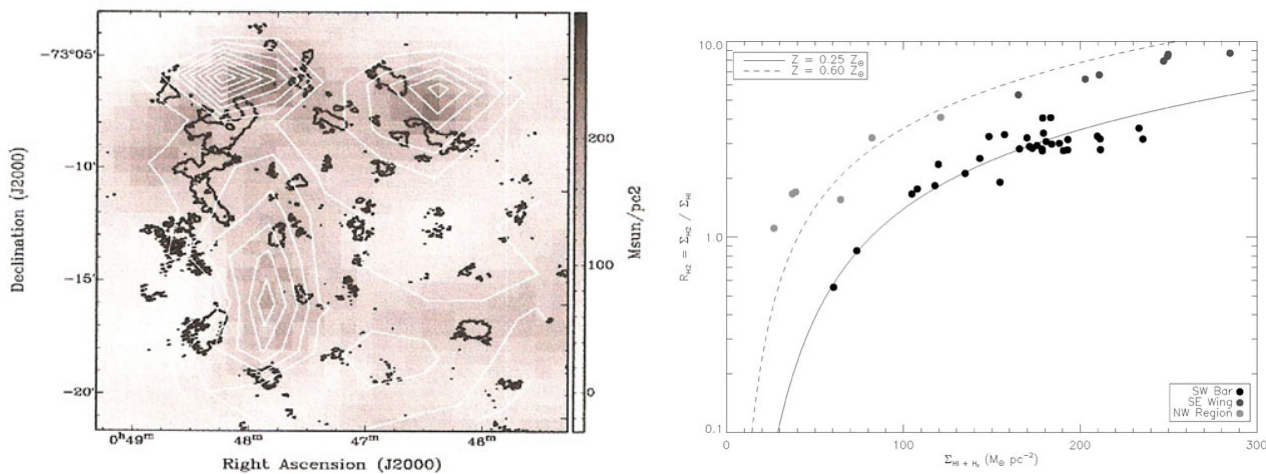

Figure 1. (Left) Selected HCRs in the southwest bar of the SMC overlaid in black contours on the $\mathrm{H}_{2}$ surface density image from Leroy et al. (2007). $\mathrm{CO}(1-0)$ emission is overlaid in white contours. HCRs are located in the outskirts of $\mathrm{CO}$ clouds but in regions with significant amount of $\mathrm{H}_{2}$. For details, see Lee et al. (2009). (Right) Molecular fraction $\left(\mathrm{R}_{\mathrm{H}_{2}}\right)$ as a function of total gas surface density $\left(\Sigma_{\mathrm{HI}+\mathrm{H}_{2}}\right)$ for HCRs. Based on the similar amounts of $\mathrm{H}$ I and $\mathrm{H}_{2}$ surface densities, HCRs most likely represent the regions where atomic-to-molecular hydrogen transition occurs in the SMC. Their molecular fraction is in agreement with the theoretical prediction from Krumholz et al. (2009) for metallicity of $1 / 4 \sim 1 / 2 \mathrm{M}_{\odot}$.

\section{References}

Krumholz, M. R., McKee, C. F., \& Tumlinson, J. 2009, ApJ, 693, 216

Lee et al., 2009, AJ, 138, 1101

Leroy et al., 2007, ApJ, 658, 1027 